\title{
POLYNOMIALS OF BINOMIAL TYPE AND LUCAS' THEOREM
}

\author{
DAVID GOSS
}

\begin{abstract}
We present various constructions of sequences of polynomials satisfying the Binomial Theorem in finite characteristic based on the theory of additive polynomials. Various actions on these constructions are also presented. It is an open question whether we then have accounted for all sequences in finite characteristic which satisfy the Binomial Theorem.
\end{abstract}

\section{INTRODUCTION}

Inspired by classical work on $p$-adic measure theory, and, in particular, the connection with the Mahler expansion of continuous functions, we discussed measure theory in finite characteristics in Go1. In characteristic $p$, an analog of the Mahler expansion was given by Wagner [Wa1] using the basic Carlitz polynomials (see Definition [6 below). By construction, the Carlitz polynomials, which are created out of additive functions via digit expansions, satisfy the Binomial Theorem as a consequence of Lucas' famous congruence (see Theorem 21). This then allowed the author, together with Greg Anderson, to compute the associated convolution measure algebra which is then isomorphic to the algebra of formal divided power series (Definition 2 below), as opposed to the ring of formal power series classically given by Mahler's result.

Inspired further by the present work of Nguyen Ngoc Dong Quan [DQ1, we have recently revisited our results from the viewpoint of the umbral calculus and the theory of sequences of polynomials satisfying the Binomial Theorem. It is indeed quite remarkable that the classical generating function of such a sequence (see Subsection 2.2) is also an element of the algebra of divided power series.

In this note we show how the Carlitz construction allows us to obtain a very large subspace of sequences satisfying the Binomial Theorem. We also show that this space is closed under multiplication in the algebra of divided power series. Moreover, still using additive polynomials, we are able to construct many other examples of sequences satisfying the Binomial Theorem which do not arise from the Carlitz method; indeed, this second construction appears to be largely complimentary to that of Carlitz. We discuss various actions on the space of divided elements and how they relate to our constructions. Along the way, we derive a decomposition of the divided power series associated to Dirac measures (see Remarks 3 below). It is now an open and very interesting question whether we then have constructed enough sequences of binomial type out of additive functions to generate all of them.

Finally, in Remarks 6, we present an umbral construction of the Carlitz module due to F. Pellarin.

We thank Nguyen Ngoc Dong Quan, Federico Pellarin, and Rudy Perkins for their help in the preparation of this note.

Date: December 10, 2014. 


\section{General Theory}

2.1. Basic notions. Let $F$ be a field and let $F[x]$ be the polynomial ring in one indeterminate over $F$. Let $P:=\left\{p_{i}(x)\right\}_{i=0}^{\infty}$ be a sequence of polynomials.

Definition 1. We say that $P$ satisfies the Binomial Theorem if and only if $p_{n}(x+y)=$ $\sum_{i=0}^{n}\left(\begin{array}{c}n \\ i\end{array}\right) p_{i}(x) p_{n-i}(y)$, for all $n \geq 0$. We let $\mathfrak{B}$ be the set of all sequences satisfying the Binomial Theorem.

Obviously $\left\{x^{i}\right\}_{i=0}^{\infty} \in \mathfrak{B}$ as does the trivial sequence $P_{0}:=\{0\}_{i=0}^{\infty}$. Another standard, nontrivial, example is the sequence $\left\{(x)_{n}\right\}$ where $(x)_{n}$ is the Pochhammer symbol defined as $(x)_{n}:=x(x-1)(x-2) \cdots(x-n+1)$. For more, we refer the reader to [RT1].

I thank Nguyen Ngoc Dong Quan for the proof of the following simple result.

Proposition 1. Let $P=\left\{p_{i}(x)\right\}_{i=0}^{\infty} \in \mathfrak{B}$ be nontrivial. Then $p_{0}(x) \equiv 1$.

Proof. Assume first that $p_{0}(x)$ is not identically 0 . Note that, by definition, $p_{0}(x+y)=$ $p_{0}(x) p_{0}(y)$. Upon setting $y=0$ we obtain $p_{0}(x)=p_{0}(x) p_{0}(0)$. By assumption, $p_{0}(x)$ is nonzero for some $x$; thus $p_{0}(0)=1$. On the other hand, $p_{0}(x-x)=p_{0}(x) p_{0}(-x)=p_{0}(0)=1$; as $p_{0}(x)$ is a polynomial, we conclude conclude that it is constant. Thus $p_{0}(x)$ is identically 1.

It remains to conclude that $p_{0}(x)$ is not identically 0 . But if it does identically vanish, a simple use of the Binomial Theorem and induction, establishes that all $p_{i}(x)$ are also trivial contradicting our assumption.

We let $\mathfrak{B}^{*} \subset \mathfrak{B}$ be the subset of nontrivial sequences.

2.2. The generating function. Let $\left\{\mathfrak{D}_{i}\right\}_{i=0}^{\infty}$ be the (nontrivial) divided power symbols with the property that

$$
\mathfrak{D}_{j} \cdot \mathfrak{D}_{j}:=\left(\begin{array}{c}
i+j \\
j
\end{array}\right) \mathfrak{D}_{i+j} .
$$

Thus in characteristic 0 one may view $\mathfrak{D}_{i}$ as $x^{i} / i$ ! and in all characteristics one can view $\mathfrak{D}_{i}$ as the divided differential operator given by

$$
\mathfrak{D}_{i} x^{n}:=\left(\begin{array}{c}
n \\
i
\end{array}\right) x^{n-i} .
$$

Definition 2. Let $R$ be a commutative ring with unit. We let $R\{\{\mathfrak{D}\}\}$ be the commutative ring of formal series $\sum a_{i} \mathfrak{D}_{i}$ with the obvious addition and multiplication.

We topologize $R\{\{\mathfrak{D}\}\}$ by using the descending chain of ideals $M_{i}=\left\{\mathfrak{D}_{i}, \mathfrak{D}_{i+1} \cdot \cdots\right\}$ for which it is complete.

Now let $H=\left\{h_{i}(x)\right\}$ be an arbitrary sequence of polynomials in $F[x]$.

Definition 3. We set

$$
f_{H}(x):=\sum_{i=0}^{\infty} h_{i}(x) \mathfrak{D}_{i} \in F[x]\{\{\mathfrak{D}\}\} .
$$

The following result is well-known and the proof follows immediately from the definitions. 
Proposition 2. We have

$$
f_{H}(x+y)=f_{H}(x) f_{H}(y)
$$

if and only if $H \in \mathfrak{B}$.

Let $\mathfrak{B} \mathfrak{D}^{*}$ be the set $\left\{f_{P}(x)\right\}_{P \in \mathfrak{B}^{*}}$; in this case, by Proposition 1, we know that $f_{P}(x)=$ $1+\{$ higher terms in $\mathfrak{D}\}$.

Theorem 1. The set $\mathfrak{B} \mathfrak{D}^{*}$ forms an abelian group under multiplication.

Proof. Let $W=\left\{w_{i}(x)\right\}$ where $w_{0}(x)=1$ and the rest vanish. Clearly $W \in \mathfrak{B}^{*}$ and $f_{W}(x)=$ $1 \in F[x]\{\{\mathfrak{D}\}\}$. Now let $P, H$ be two members of $\mathfrak{B}^{*}$ and let $g(x):=f_{P}(x) f_{H}(x)$; notice that clearly the coefficients of $g$ are polynomials in $x$. Note further that the commutativity of $F[x]\{\{\mathfrak{D}\}\}$ immediately implies that $g(x+y)=g(x) g(y)$, which shows that $\mathfrak{B D}^{*}$ is closed under multiplication.

It remains to show that every element $f_{P}(x)$ in $\mathfrak{B D}^{*}$ is invertible. As $f_{0}(x) \equiv 1$; we can write $f_{P}(x)=1+\hat{f}_{P}(x)$. Expanding $1 /\left(1+\hat{f}_{P}(x)\right)$ by the geometric series, which converges in the topology of $F\{\{\mathfrak{D}\}\}$, gives an element with polynomial coefficients inverse to $f_{P}(x)$

Remarks 1. The classical theory of polynomials of binomial type has applications in fields as diverse as combinatorics and even quantum field theory (see, for instance, [Ki1]).

\section{The Theory in finite Characteristic and Lucas' Theorem}

For the rest of this paper we assume that $F$ has characteristic $p>0$.

3.1. Lucas' Theorem. Let $q=p^{\lambda}$ where $\lambda \geq 1$. Let $m=\sum m_{i} q^{i}$ and $n=\sum n_{j} q^{j}$ be two integers written $q$-adically. Suppose $m \geq n$.

Theorem 2. (Lucas) We have

$$
\left(\begin{array}{c}
m \\
n
\end{array}\right) \equiv \prod_{i}\left(\begin{array}{c}
m_{i} \\
n_{i}
\end{array}\right) \quad(\bmod p) .
$$

Proof. By definition we have

$$
(x+y)^{m}=\sum_{e=0}^{m}\left(\begin{array}{l}
m \\
e
\end{array}\right) x^{e} y^{m-e} .
$$

But in characteristic $p$ we also have

$$
(x+y)^{m}=\prod_{i}\left(x^{q^{i}}+y^{q^{i}}\right)^{m_{i}} .
$$

The result follows upon completing the multiplication, equating 6 and 7 , and noting the uniqueness of the $q$-adic expansions.

3.2. First Results in Finite Characteristic. Let $f(x) \in F[x]$. Recall that $f(x)$ is additive if and only if $f(x+y)=f(x)+f(y)$ for all $x$ and $y$ in the algebraic closure of $F$. It is simple to see that this happens if and only if $f(x)$ only contains monomials of the form $c x^{p^{j}}$.

Our first result gives a basic connection between elements $P \in \mathfrak{B}$ and additive polynomials.

Proposition 3. Let $P=\left\{p_{i}(x)\right\} \in \mathfrak{B}$. Then $p_{p^{j}}(x)$ is additive for all $j \geq 0$.

Proof. In the Binomial Theorem all the lower terms are congruent to $0 \bmod p$ so that the result follows immediately from the definition of $P$. 
Proposition 4. Let $g(x)=1+\{$ higher terms $\} \in F[x]\{\{D\}\}$. Then $g(x)^{p} \equiv 1$.

Proof. This is an immediate consequence of Lucas' Theorem 2.

Corollary 1. The abelian group $\mathfrak{B D}^{*}$ is naturally an $\mathbb{F}_{p}$-vector space.

Remarks 2. Let $f_{P}(x) \in \mathfrak{B D *}$. By Proposition 2 we have

$$
f_{P}(p x)=f_{P}(0)=1=f_{P}(x)^{p},
$$

giving another proof of the proposition in this case.

3.3. The Carlitz Construction. Let $q=p^{\lambda}$ as before and assume that $\mathbb{F}_{q} \subseteq F$. Carlitz turned Lucas' Theorem around to construct certain sequences (Definition 6 below) satisfying the Binomial Theorem which we generalize in this subsection.

Let $E:=\left\{e_{j}(x)\right\}_{j=0}^{\infty}$ be a sequence of $\mathbb{F}_{q}$-linear polynomials (so each $e_{j}(x)$ is a finite linear combination of monomials of the form $\left.x^{q^{n}}\right)$. Let $\mathfrak{L}_{q}$ be the set of such sequences; note that $\mathfrak{L}_{p}$ is obviously an $F$-vector space. Let $i$ be a nonnegative integer written $q$-adically as $\sum_{t=0}^{m} i_{t} q^{t}$.

Definition 4. Let $E \in \mathfrak{L}_{q}$ as above. We set

$$
p_{E, i}(x):=\prod_{t} e_{t}(x)^{i_{t}}
$$

Let $P_{E}$ be the sequence $\left\{p_{E, i}(x)\right\}$. The next proposition is then basic for us.

Proposition 5. The sequence $P_{E} \in \mathfrak{B} \mathfrak{D}^{*}$.

Proof. We need to compute $p_{E, i}(x+y)=\prod_{t} e_{t}(x+y)^{i_{t}}=\prod_{t}\left(e_{t}(x)+e_{t}(y)\right)^{i_{t}}$. The result now precisely follows upon multiplying out and using Lucas' Theorem 2 .

Proposition 5 is our first construction in characteristic $p$ of polynomials satisfying the Binomial Theorem.

Example 1. For $i \geq 0$ let $\ell_{q}(i)$ be the sum of its $q$-adic digits. Then the sequence $\left\{x^{\ell_{q}(i)}\right\}$ of elements in $F[x]$ satisfies the Binomial Theorem. Indeed, this is just Proposition 5 where all $e_{t}(x)=x$.

3.4. The Connection with Nonarchimedean Measure Theory. Let $A:=\mathbb{F}_{q}[\theta]$ and, for $t \geq 0$ an integer, let $A(t):=\{a \in A \mid \operatorname{deg}(a)<t\}$; notice that $A(t)$ is an $\mathbb{F}_{q}$-vector space of dimension $t$. In this subsection $F$ will be a field containing $A$.

Definition 5. Let $t \geq 0$ be an integer. We let $D_{t}$ be the product of all monic elements of degree $t$ and

$$
e_{t}(x):=\prod_{a \in A(t)}(x-a) .
$$

It is easy to see that $e_{t}(x)$ is additive (indeed, $\mathbb{F}_{q}$-linear). We set $E:=\left\{e_{t}(x) / D_{t}\right\}_{t=0}^{\infty}$, and now let $i=\sum_{t=0}^{m} i_{t} q^{t}$ be written $q$-adically as above.

Definition 6. We define the Carlitz polynomials $P_{E}=\left\{p_{E, i}(x)\right\}$ by

$$
p_{E, i}(x):=\prod_{t}\left(\frac{e_{t}(x)}{D_{t}}\right)^{i_{t}},
$$

where $e_{t}(x)$ is given in Equation 9 . 
It is traditional to set $G:=P_{E}$ and $G_{i}(x):=p_{E, i}(x)$. Carlitz has shown that $G_{i}(a) \in A$ for all $a \in A$ and by Proposition 5 we know that $G$ satisfies the Binomial Theorem. For a nontrivial prime $\mathfrak{v}$ of $A$ we let $A_{\mathfrak{v}}$ be the completion of $A$ at $\mathfrak{v}$. C. Wagner [Wa1] has shown that $G$ forms an orthonormal Banach basis for the space of continuous functions from $A_{\mathfrak{v}}$ to itself, see also Co1. Using the fact that $G$ satisfies the Binomial Theorem, the author, and Greg Anderson, noticed that this implies that the convolution algebra of Nonarchimedean measures on $A_{\mathfrak{v}}$ with values in $A_{\mathfrak{v}}$ is then isomorphic to $A_{\mathfrak{v}}\{\{\mathfrak{D}\}\}$; see [Go1].

Example 2. Let $\alpha \in A_{\mathfrak{v}}$. The Dirac measure at $\alpha, \delta_{\alpha}$, is defined by $\int_{A_{\mathfrak{v}}} f(x) d \delta_{\alpha}(x)=f(\alpha)$ for all continuous $f(x)$ (note that in non-Archimedean analysis this construction gives a true, bounded, measure). We know that $f(x)$ can be expressed as $\sum c_{i} G_{i}(x)$ for a unique sequence $\left\{c_{i}\right\}$ of scalars which tends to 0 as $i \rightarrow \infty$. Thus, we have $\delta_{\alpha}$ corresponds to $\sum_{i} G_{i}(\alpha) \mathfrak{D}_{i} \in A_{\mathfrak{v}}\{\{\mathfrak{D}\}\}$. Moreover, by definition, the convolution of $\delta_{\alpha}$ and $\delta_{\beta}$ is $\delta_{(\alpha+\beta)}$, and this precisely corresponds to Proposition 2 in the description of measures as elements of $A_{\mathfrak{v}}\{\{\mathfrak{D}\}\}$.

3.5. The Carlitz Construction is closed under Multiplication of Generating Functions. We now return to having $F$ be an arbitrary field with $\mathbb{F}_{q} \subset F$. By Corollary 1, the group $\mathfrak{B D}^{*}$ is an $\mathbb{F}_{p}$-vector space.

Definition 7. We let $\mathfrak{B} \mathfrak{D}_{q, 0}^{*} \subseteq \mathfrak{B} \mathfrak{D}^{*}$ be the subset of those generating functions that arise from $\mathfrak{L}_{q}$ by the Carlitz construction Proposition 5 .

Let $W=\left\{w_{t}\right\}$ and $V=\left\{v_{t}\right\}$ be two elements of $\mathfrak{L}_{p}$ with sum $W+V$. Let $P_{W}, P_{V}$ and $P_{(W+V)}$ be the corresponding sequences of polynomials satisfying the Binomial Theorem with $f_{P_{W}}(x), f_{P_{V}}(x)$ and $f_{P_{(W+V)}}(x)$ the corresponding generating functions.

Theorem 3. In $F[x]\{\{D\}\}$ we have

$$
f_{P_{W}}(x) \cdot f_{P_{V}}(x)=f_{P_{(W+V)}}(x) .
$$

Proof. Let $i=\sum_{t} i_{t} q^{t}$ be written $q$-adically as before. By definition

$$
p_{(W+V), i}(x)=\prod_{t}\left(w_{t}(x)+v_{t}(x)\right)^{i_{t}} .
$$

One now expands out and uses Lucas as before. The result is exactly the element obtained by multiplying $f_{P_{W}}(x)$ and $f_{P_{V}}(x)$ and the result follows.

Corollary 2. $\mathfrak{B} \mathfrak{D}_{q, 0}^{*} \subseteq \mathfrak{B} \mathfrak{D}^{*}$ is an $\mathbb{F}_{p}$-subspace.

Proof. The space $\mathfrak{B} \mathfrak{D}_{q, 0}^{*}$ is the image of $\mathfrak{L}_{p}$ under the linear injection $E \mapsto P_{W}$.

Corollary 3. Let $E \in \mathfrak{L}_{q}$ and let $-E$ be its inverse obtained by negating all its elements. Then

$$
f_{P_{E}}(x) \cdot f_{P_{-E}}(x)=1 .
$$

Example 3. Returning to the case of Example 2, the measure theoretic version of Corollary 3 is precisely the standard fact that the convolution of $\delta_{\alpha}$ and $\delta_{-\alpha}$ is $\delta_{0}=1$.

Remarks 3. Theorem 3 implies that all elements of $\mathfrak{B} \mathfrak{D}_{q, 0}^{*}$ may be expressed as a, possibly infinite, convergent product over those elements created out of only one nonzero additive function. In particular, we derive such a product decomposition for the Dirac measures $\delta_{\alpha}$ as elements of $A_{\mathfrak{v}}\{\{\mathfrak{D}\}\}$. In fact, when $\alpha$ is not an element of $A$, this product decomposition has 
infinitely many terms. In turn, this product decomposition corresponds measure theoretically to expressing $\delta_{\alpha}$ as an "infinite convolution," a concept which is well-known in classical measure theory, see for example [EST].

\section{A Second Construction}

4.1. A Counterexample. Corollary 2 states that $\mathfrak{B} \mathfrak{D}_{q, 0}^{*}$ is a subspace of $\mathfrak{B} \mathfrak{D}^{*}$. In the next example we will produce an example of an element of $\mathfrak{B D}^{*}$ not lying in $\mathfrak{B D}_{q, 0}^{*}$ for any $q$.

Example 4. Let $\left\{f_{i}(x)\right\}_{i=1}^{\infty}$ be a collection of non-trivial additive functions. Let $f(x)=$ $1+\sum_{i=0}^{\infty} f_{i}(x) \mathfrak{D}_{p^{i}-1}$. Then $f(x) \in \mathfrak{B D}^{*} \backslash \mathfrak{B D}_{q, 0}^{*}$. Indeed, $f(x+y)=f(x) f(y)$ by the additivity of the $f_{i}(x)$ and the fact that for $i, j>0, \mathfrak{D}_{p^{i}-1} \cdot \mathfrak{D}_{p^{j}-1}=0$ (due to the vanishing of the binomial coefficient); thus $f(x) \in \mathfrak{B} \mathfrak{D}^{*}$ by Proposition 2. As the coefficients of $\mathfrak{D}_{p^{t}}$, $t>0$, vanish, it can not be in $\mathfrak{B D}_{q, 0}^{*}$ for any $q$.

Remarks 4 . It is easy to see that the element $f(x)$ of the above example can be expressed as the infinite product $\prod_{i}\left(1+f_{i}(x) \mathfrak{D}_{p^{i}-1}\right)$. Note also that multiplying $f$ by an element of $\mathfrak{B} \mathfrak{D}_{p, 0}^{*}$ also can not lie in $\mathfrak{B} \mathfrak{D}_{p, 0}^{*}$ as it is a group.

4.2. A Second Construction of Elements in $\mathfrak{B D}^{*}$. Example 4 leads to a very general construction of elements of $\mathfrak{B D}^{*}$ which is, in some sense, complimentary to the Carlitz construction of Subsection 3.3.

Definition 8. We say a sequence $X:=\left\{\mathfrak{D}_{i_{j}}\right\}_{j=0}^{\infty}$ of divided elements is a null sequence if and only if $\mathfrak{D}_{i_{j}} \cdot \mathfrak{D}_{i_{t}}=0$ for all $j$ and $t$ (where $j=t$ is permitted).

Now let $E:=\left\{e_{j}(x)\right\}_{j=0}^{\infty} \in \mathfrak{L}_{p}$ be a sequence of additive functions and define

$$
f_{X, E}(x)=1+\sum_{j} e_{j}(x) \mathfrak{D}_{i_{j}} .
$$

Proposition 6. The element $f_{X, E}(x) \in \mathfrak{B} \mathfrak{D}^{*}$.

Proof. This follows exactly as in Example 4.

Note, as before, we readily deduce the convergent product

$$
f_{X, E}(x)=\prod_{j}\left(1+e_{j}(x) \mathfrak{D}_{i_{j}}\right) .
$$

Let $\pi_{X}: X \rightarrow \mathfrak{B D} \mathfrak{D}^{*}$ be defined by $\pi_{X}(E):=f_{X, E}(x)$. The next result then follows immediately.

Proposition 7. The mapping $\pi_{X}$ is an injection of $\mathfrak{L}_{p}$ into $\mathfrak{B} \mathfrak{D}^{*}$.

4.3. The Action of the Group $S_{(q)}$. In Go2] we introduced the group $S_{(q)}$ of homeomorphisms of $\mathbb{Z}_{p}$ to itself. The construction of the group is very simple; let $\sigma$ be a permutation of the set $\{0,1, \ldots\}$ and let $y \in \mathbb{Z}_{p}$ be written $q$-adically as $\sum_{i=0} y_{i} q^{i}$. One then defines $\sigma_{*} y=\sum_{i} y_{i} q^{\sigma i}$. Note that this action preserves the positive integers. Furthermore, we established that, in characteristic $p>0$, the induced mapping $\sigma_{*}\left(\mathfrak{D}_{j}\right):=\mathfrak{D}_{\sigma_{*} j}$ is an automorphism of $R\{\{\mathfrak{D}\}\}$.

Proposition 8. The automorphism $\sigma_{*}$ stabilizes both $\mathfrak{B} \mathfrak{D}^{*}$ and $\mathfrak{B} \mathfrak{D}_{q, 0}^{*}$. 
Proof. Since $\sigma_{*}$ is a ring homomorphism, the first statement follows from Proposition 2. The second statement follows because $\sigma_{*}$ merely changes the order of the additive functions in the Carlitz construction which is inessential.

Corollary 4. Let $f(x)$ be the function of Example 4 and let $f^{\sigma}$ be its image under $\sigma_{*}$. Then $f^{\sigma} \in \mathfrak{B D}^{*} \backslash \mathfrak{B} \mathfrak{D}_{q, 0}^{*}$.

Remarks 5. Let $X=\left\{\mathfrak{D}_{i_{j}}\right\}$ be as in Subsection 4.2 and let $X^{\sigma}=\left\{\mathfrak{D}_{\sigma_{*} i_{j}}\right\}$. Then clearly $\sigma_{*} \circ \pi_{X}=\pi_{X^{\sigma}}$.

4.4. Further Actions. Federico Pellarin has kindly pointed out three other actions on the algebra $R\{\{\mathfrak{D}\}\}$ in finite characteristic.

Definition 9. Let $f=\sum a_{i} \mathfrak{D}_{i}$.

1. We set $\pi_{1}(f):=\sum a_{i}^{p} \mathfrak{D}_{I}$.

2. We set $\pi_{2}(f):=\sum a_{i} \mathfrak{D}_{p i}$.

3. Let $r \in R$. We set $\pi_{3}(f):=\sum a_{i} r^{i} \mathfrak{D}_{i}$ (the "evaluation" map).

It is easy to see that all three of these maps are endomorphisms of $R\{\{\mathfrak{D}\}\}$ with the first being an obvious automorphism in the case where $R$ is a perfect field. They therefore take $\mathfrak{B D} \mathfrak{D}^{*}$ to itself. It is also straightforward to see that these endomorphisms stabilize $\mathfrak{B D}_{p, 0}^{*}$ and that the $q$-th power of the first two actions stabilizes $\mathfrak{B} \mathfrak{D}_{q, 0}^{*}$. Their induced actions on the maps $\pi_{X}$ of Subsection 4.2 are also easy to compute.

4.5. Final Remarks. We have seen that both the Carlitz construction (Corollary 2) and our second construction in Subsection 4.2 give rise to injections of the spaces $\mathfrak{L}_{q}$ into $\mathfrak{B} \mathfrak{D}^{*}$.

Question 1. Do the images of the above injections generate $\mathfrak{B} \mathfrak{D}^{*}$ ?

In other words, do the additive polynomials ultimately account for all elements of $\mathfrak{B}$ ? Note that in characteristic 0, the elements of $\mathfrak{B}$ are described by the Bell Polynomials [RT1].

Remarks 6. The umbral theory of [RT1], with its "black magic" of linear maps etc., has further connections with the arithmetic of function fields as pointed out by F. Pellarin and which we briefly describe here. Let $C$ be the Carlitz module and let $\omega(t)$ be the AndersonThakur function as in [Pe1]. Let $\tau$ be the $q$-th power mapping acting as in [Pe1] where one defines (Definition 2.6) the polynomials $b_{j}(t)$ by $\tau^{j} \omega(t)=b_{j}(t) \omega(t)$. It is readily seen that $b_{j}(t)=\prod_{e=0}^{j-1}\left(t-\theta^{q^{e}}\right.$ ) (and in fact, as shown in ibid, these polynomials are universal in that the coefficients of both the Carlitz exponential and logarithm may be easily expressed using them). Now define the $A$-linear map from $A[\tau] \rightarrow A[t]$ by $\tau^{i} \mapsto b_{i}(t)$. This gives an isomorphism with the inverse given by $t^{j} \mapsto C_{\theta^{J}}$ as one deduces from the theory of $\omega(t)$. In particular, we derive another construction of the Carlitz module and another indication of its ubiquity.

\section{REFERENCES}

[Co1] K. ConRad: The digit principle, J. Number Th. (2000) 230-257.

[DQ1] N.N. Dong Quan: The classical Umbral Calculus, and the Flow of a Drinfeld module, arXiv:1405.2135.

[EST] C. Escribano, M.A. Sastre, E. Torrano: Moments of infinite convolutions of symmetric Bernoulli distributions, it J. Comp. and Applied Math. (2003) 191-199.

[Go1] D. Goss: Fourier Series, Measures and Divided Power Series in the Theory of Function Fields, K-Theory 1 (1989) 533-555. 
[Go2] D. Goss: Zeta-Phenomenology, in: Noncommutative geometry, arithmetic, and related topics Johns Hopkins Press (2011) 159-182.

[Ki1] V.V. KISIL: Polynomial Sequences of Binomial Type and Path Integrals, Ann. Comb. (2002) 45-56.

[Pe1] R. Perkins: Explicit formulae for $L$-values in positive characteristic, Math. Z. (2014) 279-299.

[RT1] G.-C. Rota, B.D. TAYLOR: An introduction to the umbral calculus, in: Analysis, geometry and groups: a Riemann legacy volume, Hadronic Press, Palm Harbor, FL (1993) 513-525.

[Wa1] C. WAGNER: Interpolation series for continuous functions on $\pi$-adic completes of GF $(q, x)$, Acta Arith. 17 (1971) 389-406.

Department of Mathematics, The Ohio State University, $231 \mathrm{~W} .18^{\text {th }}$ Ave., Columbus, Ohio 43210

E-mail address: dmgoss@gmail.com 